\title{
Marketing Ecológico
}

\author{
Ecological Marketing
}

\section{RESUMEN}

Muchos expertos en el marketing perciben que este mundo globalizado, lo que define es el valor agregado del producto. Señal que no solo es de juicio técnico, sino que se encuentra en la conciencia de los consumidores: estos conocen que el producto o servicio no es suficiente, ellos necesitan algo más, están buscando un factor que los diferencie, que, al encontrarlo, estos lo premian. En general los consumidores se encuentran buscando un plus o beneficio para su grupo sean estos sociales, familiares, etc., El marketing responsable obedece a las expectativas y sale como una de las aristas de la RSE (Responsabilidad Social Empresarial).

Para rehusar los envases y embalajes se estipula tres características especiales: uno el transporte industrial, como es el uso de los palets. Dos en la reparación reacondicionamiento y reutilización de productos como la idea de recuperar el producto y hacerlo apto para la venta, el objetivo del reciclado es el uso de residuos como alternativa de materias primas, considerada como responsabilidad del marketing de productos; y tres las estrategias de transformación de materiales como la conversión en energía y el compost.

Palabras claves: Estrategias de marketing ecológico; canales inversos para reusar envases y embalajes.

\begin{abstract}
Marketing specialists know that in a globalized world what defines is the added value of the product. This premise is not only of technical knowledge but it is also set on the mind of the consumers: they know that the services or the products are not enough; they want something else, they look for a differentiating factor and if they find it, they reward it. Consumers are in the search of a plus or benefit for their family and social group, responsible marketing meets these expectations and emerges as one of the aspects of RSE.
\end{abstract}

\section{Ricardo Rolando Fonseca Saldaña ${ }^{1}$ \\ rfonseca20@yahoo.es}

Adán H. Estela Estela ${ }^{2}$ adan.estela22@gmail.com Universidad Ricardo Palma

\footnotetext{
${ }^{1}$ Doctor en Administración. Magister en Turismo y Hotelería con mención en Marketing Turístico y Hotelero. Título Profesional de Economista. Representante de Docentes Categoría Asociado al Consejo de Facultad de Ciencias Económicas y Empresariales - URP Jefe de la Oficina de Grados y Títulos de la Facultad de Ciencias Económicas y Empresariales - URP. Catedrático en la Universidad Ricardo Palma en la Facultad de Ciencias Económicas y Empresariales; de la Escuela Profesional de Turismo, Hotelería y Gastronomía. Catedrático en la Universidad Nacional Mayor de San Marcos de la Facultad de Ciencias Administrativas; de la Escuela Profesional de Administración de Turismo. Experiencia en Empresas del sector privado. Asesor y Consultor en Empresas de Servicios en Administración Comercial, Ventas y Marketing. Profesor Investigador

${ }^{2}$ Doctor en Administración. Maestría en Educación. Profesor Universitario URP. UNMSM. Profesor en el Doctorado y Maestría UNMSM y URP. Empresario dedicado en las Artes Gráficas. Profesor Investigador. Asesor y Consultor de Empresas
}

(C) Los autores. Este artículo es publicado por Gestión en el Tercer Milenio de la Facultad de Ciencias Administrativas de la Universidad Nacional Mayor de San Marcos. Este es un artículo de acceso abierto, distribuido bajo los términos de la licencia Creative Commons Atribucion - No Comercia_Compartir Igual 4.0 Internacional. (http://creativecommons.org/licenses/by-nc-sa/4.0/) que permite el uso no comercial, distribución y reproducción en cualquier medio, siempre que la obra original sea debidamente citada. 
To reuse containers and packaging three special characteristics are stipulated. One, industrial transportation, as for example the use of pallets. Two: in repairing, reconditioning and reutilizing products, the idea is recuperating the product and making it available for sale; the objective of recycling is the use of remnants as an alternative to raw materials, which is considered responsibility of the marketing of products, and three. The strategies of transformation of materials as for instance the conversion into energy and the compost.

Keywords: Strategies in ecological marketing; inverse channels to reuse containers and packaging.

\section{INTRODUCCIÓN}

Las repercusiones actuales del medio ambiente determinan que el trabajo sea a cerca de la relevancia que tiene el marketing ecológico para reorientar al consumidor en su elección, así como en la organización a reorganizar en el comportamiento empresarial con el solo propósito de cuidar el medio ambiente. Por lo tanto, expondremos sobre la conceptualización, las estrategias del marketing ecológico y sobre los canales inversos para el reciclado.

\section{OBJETIVO DEL ENSAYO}

Dicho trabajo tiene como objetivo dar a conocer y aplicar conceptos, además de las diferentes estrategias que presenta el marketing ecológico en cada sector y segmentos de mercado

\section{ARGUMENTACIÓN \\ El Marketing Ecológico}

El Marketing Ecológico llamado también Marketing verde. La American Marketing Associaciation define "Green Marketing" como la comercialización de productos seguros para el medio ambiente. Asimismo, esta actividad concentra operaciones como: reformas al producto, durante el proceso de producción, las novedades en el packaging y en la comunicación. La palabra ecología literalmente "Ökologie del alemán «oikos», «casa, vivienda, hogar», y logia, «tratado o estudio» fue empleada por primera vez por Ernest Haeckel. Ecología la que se encarga de estudiar la interrelación entre los organismos con su medio ambiente. Entonces se puede indicar que el Marketing ecológico tiene relación con la impresión que repercute en el contexto natural.
La palabra desarrollo sustentable referido en el Informe Brundtland (1987) llevada a cabo en la Cumbre de Río (1992) se expuso como el principio fundamental. Definida como: "el desarrollo que satisface las necesidades del presente sin comprometer las capacidades que tienen las futuras generaciones para satisfacer sus propias necesidades". (Sustentator, 2010).

Kotler define el marketing como una organización comprometida considerando los deseos e intereses de los consumidores, las necesidades de la empresa y los intereses de la sociedad a largo plazo. (Sustentator, 2010)

De estos conceptos podemos tener en cuenta lo siguiente:

- Para definir Marketing Verde está relacionado con el cuidado del medio ambiente. El impulso de nuevos productos puede resultar complicado, por la necesidad de disminuir la repercusión ambiental.

- Debemos conservar el suelo, el agua y el aire de manera solidaria. Es esencial tener en cuenta la repercusión que pueda ocurrir para las próximas generaciones, ya que en la mayoría de las veces usamos los recursos naturales como si estos fueran inagotables.

Las actividades realizadas por las empresas tienen como fin permanecer en el mercado generando beneficios. Pero las empresas ahora deben de ejecutar otras operaciones que originen un valor añadido a la entrega de una de estas actividades con referencia al marketing responsable.

Para Chiriboga (2011) El marketing responsable posiciona productos con la realidad del consumidor por dos condiciones: aumenta 
o disminuye ventas e influye en la marca. Las empresas buscaban como relacionarse con su cliente por lo que se debe tener presente la calidad, los cumplimientos, la forma en que se producen y comercializan, etc. Empresas que con las buenas prácticas en sus procesos de producción/comercialización/ comunicación instauran bondades del producto, y se distinguen del resto. (Chiriboga, 2011)

Yohn Chiriboga (2011), determina que son tres los factores que aseguran una política de mercadeo responsable:

\section{a. Satisfacción a consumidores}

Referencia a la empresa vinculada con los consumidores, recomendando mecanismos de comunicación que permitan un diálogo fluido y la retroalimentación. La tarea es incorporar la satisfacción del cliente y la manera en que la empresa recoge las opiniones y los usa para la mejora continua de sus productos, sean estos tangibles o intangibles. Así como recoger las opiniones de los consumidores bajo un sistema de acopiar las peticiones y reclamos con las características del producto.

\section{b. Política publicitaria}

Publicidad elaborada a través de razones éticas, es expresar el respeto a la integridad de las personas, sin la discriminación hacia los demás la negativa de publicidad engañosa. Por ejemplo: Elaborando un "Código de Ética Publicitaria" que incorpore características del producto. Promover valores basados en derechos humanos, contenido de publicidad clara, sencilla y fácil de comprender, Incorporando otros elementos que promuevan "Marketing social".

\section{c. Conocimiento de daños potenciales}

La labor que desarrolla la empresa debe velar por las condiciones de salud y seguridad de sus consumidores. La organización se preocupa por sus procesos de producción y que estos no causen daño al consumidor. Por ejemplo: Elaborando políticas de conocimiento y mejora en procesos, por posibles daños que pueda ofrecer el producto/servicio.

\section{Estrategias de marketing ecológico}

Siguiendo a Limonche y Peñaranda (2013) La coherencia obliga a que la estrategia ecológica de un producto o marca esté en consonancia con la propia estrategia ecológica general de la empresa y considerar, además de las políticas de ventas de la empresa, cuestiones ecológicas que afectan globalmente al consumidor, como:

- La limitación de los recursos naturales.

- Desarrollo sostenible frente a consumismo.

- La lucha contra la contaminación y por la biodiversidad.

- La minimización del impacto por los residuos mediante la reducción (minimización del envase), reutilización y el reciclado.

Todo esto se debe de compatibilizar con el objetivo empresarial de obtener una buena rentabilidad, y manteniendo la competitividad.

El primer paso dentro de la estrategia de marketing ecológico es el desarrollo de productos ecológicos, lo que lleva consigo:

- El abandono de productos y formas de producir perjudiciales para el medio ambiente.

- Abandono de envases que perjudiquen el medio ambiente.

- Minimización en el uso de energías fósiles y materias primas.

- Asunción de los costes sociales que genera la producción y el consumo de los productos.

Esto se verá compensado por la diferenciación del producto, para lo que se propone, una de las siguientes estrategias:

- Rediseño del producto existente.

- Diseño de un producto nuevo.

- Sustitución de productos ecológicamente no aceptables.

Los cambios son cada día más complejos cuando la empresa ya está en funcionamiento; pero sucede lo contrario en empresas que recién se crean. Algunos elementos para identificar el producto ecológico:

- Reducción del número de componentes del producto. 
- Reducción del consumo de energía.

- Diseño "limpio", evitando consumibles que generen polución.

- Productos reciclables y reciclados.

- Minimización de envases.

Las empresas necesitan la confianza del público en sus acciones medioambientales, por ello una estrategia de actuación puede ser el "partenariado" o asociación con las organizaciones medioambientales. Estas pueden ser: administraciones públicas, grupos medioambientales, ONG (Organización Goburnemental), organizaciones de consumidores. (Limonche \& Peñaranda, 2013)

\section{Canales inversos para reusar el envase y embalaje}

\section{a. Transporte industrial}

De acuerdo con Andrea Muñoz (2013) para el asunto de material de traslado industrial determina la existencia de empresas especializadas. Como es el caso de los alquileres de los palets por os transportistas para el traslado y entrega de los productos los que aseguran la disposición reúso mantenimiento y el destino final, soportes, protecciones, los que pueden ser de madera, plástico, metal, cartón, los que deben estar diseñados de manera ecológica para un reúso posterior empleados en un nuevo ciclo de transporte, el que determina la disminución de costes de protección de materiales.

\begin{abstract}
"Las entregas en sistemas just in time (JIT) cumplen estas especificaciones de manera aceptable, por lo que han sido empleadas estas técnicas de reúso con éxito. Pero hay otros condicionantes que las técnicas JIT tienen, como son la necesidad de una información rápida, fluida y activa entre los participantes y enfocados a una actividad compatible con esta reutilización de materiales" (Muñoz, 2013).
\end{abstract}

\section{b. Reparar, reacondicionar y reutilizar productos}

Estrategias basadas en recuperar los productos en el final del ciclo de producción para enviarlo al mercado. Lo incompatible está en la manera de recoger y de enviar al fabricante original como pueden ser en los productos de alto valor siendo estos lo ordenadores y fotocopiadoras, que mu- chas veces se ha puesto en práctica por medio de los descuentos por recompra de equipos viejos canjeados por equipos nuevos o por las operaciones de leasing. Las que sustituyen los equipos después de un periodo de tiempo de uso.

El objetivo del recicle es usar los residuos como alternativa de materia prima, proceso que tiene que ser considerado dentro del ciclo de vida responsable del márketing de productos.

\section{Marketing de recicle: causas}

El recicle radica en el recojo de materiales de manera ordenada, homogénea y tratarlos con el fin de que tengan características esenciales para ser usados en diversos procesos de producción o para su venta. Muchas veces el material reciclado compite con material nuevo como sustituto ya que si no cuenta con material requerido puede mezclarse o ser empleado para elaborar nuevos productos.

\section{Suministro continuo}

Los métodos de producción necesitan de grandes cantidades de insumos y que la escases de materias primas, provoca altos costos de producción y comercio, los que podrían ser rechazados por el mercado. Si se busca ser competitivo en precio se tendrá que recurrir al aprovechamiento de escala en el uso de material reciclado.

Residuos urbanos. El flujo de material debe estar asegurado por lo que supone que se debe relaciona con la ciudadanía y los hogares en el recojo de su basura. Siendo la separación de materiales una tarea compleja, por lo que se ha diseñado programas de selección por tipo de residuo en los hogares con la finalidad de dar facilidad al tratamiento en la planta.

Residuo industrial. Cuando las cantidades son mayores por lo general se compactan para reducir el volumen que inclusive pueden recibir un tratamiento especial revalorizando siendo lo mismo con el plástico en las empresas envasadoras que contratan a empresa especializadas para su recuperación y ofrecen a otras organizaciones como materia prima.

\section{Construcción de canales inversos}

Las tareas para este tipoi de actividad decanal inverso son: "primero, recojo y proce- 
so de gran cantidad de material". "procesar el material para conseguir la calidad deseada". "Y tercero, mantener la continuidad en los intercambios".

\section{Mercado para productos de recicle}

Para la viabilidad de un producto reciclado, debe encontrar una demanda que justifique su producción por lo que se debe colocar programas de búsqueda que permitan emplear material reciclado, sin afectar la calidad de su producción.

\section{c. Las estrategias de trasformación de materiales}

Para victoria muñoz (2013) la conversión de materiales principalmente se indica para residuos de tipo orgánico como, los restos de refrigerios, el desperdicio agrícola, la madera, los plásticos, gomas, papel no reciclable, su propiedad básica es la transformación de energía por medio de la combustión, generar abono orgánico (compost) o convertir en otras materias orgánicas por destitución.

\section{Conversión en energía}

El transformar la energía por combustión se realiza a través de irrigar el petróleo a un producto que provoque su combustión con el beneficio del calor que origine para transformarlo en energía eléctrica, o como fuente de agua caliente.

Desperdicios convertidos en cenizas, estas últimas se pueden decir que el resultado es un diez por ciento del volumen inicial, rebajando los vertederos municipales. Que debe realizarse después de las técnicas de reciclado y compost, utilizando las grandes cantidades de materiales disponibles de los residuos municipales. (Muñoz, 2013)

\section{El compost.}

Es material de desperdicio de hogares o fabricantes agrarios. Su recicle demanda tratamiento apartado de material de envases y embalajes por sus rasgos físicas, químicas y biológicas es decir de material de rápida desintegración. Se necesita de un reojo rápido por la degradación rápida de los residuos orgánicos muy rica, pero pierden sus características más útiles. El recojo municipal es la fuente grande de residuos, pero es necesaria su separación, para un proceso rápido. Se han hecho experiencias que aseguren unos resultados aceptables, con contenedores para este tipo de residuos, pero es preciso que la población esté sensibilizada y educada para dicho comportamiento, (Muñoz, 2013).

“El procesamiento posterior de los residuos es una fermentación controlada que los convierte en nutrientes y abonos orgánicos para agricultura y jardinería, lo que eleva su valor de utilización y los convierte en alternativa a los abonos químicos" (Muñoz, 2013).

\section{CONCLUSIONES}

Los especialistas de marketing saben que el valor agregado del producto lo define gracias a la impresión y comunicación premisa que está en la conciencia de los consumidores que buscan un factor diferenciador y si lo encuentran lo apremian.

El marketing ecológico o marketing verde se define como la mercadotecnia de productos seguros para el medio ambiente, este incorpora modificaciones al producto en el proceso de producción, que tiene que ver con el impacto que repercute en el entorno natural. Además, porque debemos cuidar los recursos naturales por que, muchas veces se usan como si nunca se van a terminar.

Las actividades empresariales deben generar valor a lo que entreguen, a través de actividades relacionadas con el marketing responsable, ya que este posiciona productos por dos condiciones aumenta o disminuye ventas y por otro lado influye en la marca. Así como el empleo de estrategias como las luchas contra la contaminación y protección de recurso naturales.

\section{REFERENCIAS BIBLIOGRÁFICAS}

Chiriboga, J. Y. (13 de octbubre de 2011). Marketing Responsable. Recuperado el 20 de enero de 2018, de Responsabilidad Social Corporativa: http:// responsabilidadsocialmariaandrader.blogspot. pe/2011/10/marketing-responsable.html

INTI. (2016). El valor de los residuos: Distintos modos de Reducir, Reutilizar, Reciclar y Revalorizar residuos industriales. (I. N. Industrial, Ed.) Buenos Aires, Argentina: centro INTI-Ambiente. 
Obtenido de https://www.inti.gob.ar/ambientesg/pdf/El_valor_de_los_residuos.pdf

Limonche, Y. M., \& Peñaranda, F. P. (2013). Inserción del mercadeo verde en empresas del sector papelero en Cauca y el Valle del Cauca. Tesis, Universidad Autónoma de Occidente, Santiago de Cali, Colombia. Recuperado el 10 de diciembre de 2017, de https://red.uao.edu.co/bitstream/10614/5144/1/TMD01530.pdf
Muñoz, V. S. (2013). Marketing Ecológico. Concepción, Chile. Obtenido de http://www.victoria-andrea-munoz-serra.com/MARKETING/ marketing_\%20ecologico.pdf

Sustentator. (09 de abril de 2010). ¿Qué significa "Marketing Verde"? Recuperado el 15 de febrero de 2018, de http://www.sustentator.com/bloges/2010/04/qu-significa-marketing-verde/ 